\title{
Surgery and Neurointervention for Intracranial Atherosclerotic Steno-occlusive Disease
}

\author{
Sepideh Amin-Hanjani, ${ }^{1}$ Simon Chun Ho Yu, ${ }^{2}$ Fady Charbel, ${ }^{1}$ Joji Inamasu, ${ }^{3}$ Yoko Kato ${ }^{3}$ and George Kwok-Chu Wong ${ }^{4}$ \\ 1. Professor, Department of Neurosurgery, University of Illinois at Chicago, Chicago, Illinois, US; 2. Professor, Department of Imaging and Interventional Radiology, \\ Chinese University of Hong Kong, Hong Kong; 3. Professor, Department of Neurosurgery, Fujita Health University, Toyoake, Japan; \\ 4. Professor, Division of Neurosurgery, Chinese University of Hong Kong, Hong Kong
}

\begin{abstract}
Neurosurgeons and neurointerventionists interested in cerebral revascularisation to prevent stroke from intracranial atherosclerotic steno-occlusive disease were disappointed in 2011 with the closure of two important negative studies: the Carotid Occlusion Surgery Study (COSS) and Stenting and Aggressive Medical Therapy for Preventing Recurrent Stroke in Intracranial Stenosis (SAMMPRIS) study. Debates are centred on what causes these failures. While extracranial-intracranial (EC-IC) bypass and neurointervention (angioplasty and/ or stenting) cannot be considered a routine intervention for patients presenting with initial ischaemic event in the setting of atherosclerotic steno-occlusive disease, selected patients with severe haemodynamic impairment and/or recurrent symptoms despite maximal medical therapy may still benefit from surgery and neurointervention at high-volume centres, which can offer the procedure with low perioperative morbidity.
\end{abstract}

\section{Keywords}

Angioplasty, cerebral ischaemia, EC-IC bypass, stenting, stroke

Disclosure: The authors have no conflicts of interest to declare.

Received: 5 August 2013 Accepted: 31 October 2013 Citation: European Neurological Review, 2013;8(2):170-4 DOI:10.17925/ENR.2013.08.02.170

Correspondence: George Kwok-Chu Wong, Professor (Clinical), Division of Neurosurgery, Department of Surgery, 4/F Clinical Science Building, Prince of Wales Hospital, 30-32 Ngan Shing Street, Shatin, New Territories, Hong Kong SAR China. E: georgewong@surgery.cuhk.edu.hk

\section{Surgery for Occlusive Atherosclerotic Disease} Extracranial-intracranial (EC-IC) bypass for revascularisation in the setting of atherosclerotic occlusive disease has remained a topic of intense interest and scrutiny over the last four decades. The underlying premise of EC-IC bypass in this setting is to provide blood flow augmentation in the setting of cerebral ischaemia, aiming to improve blood flow to oligemic brain tissue.

\section{Evidence for Extracranial-Intracranial Bypass Anterior Circulation}

In evaluating the efficacy of EC-IC bypass for ischaemia, the primary focus has been on anterior circulation disease. Flow augmentation bypass in the anterior circulation primarily entails a bypass between the superficial temporal artery and the middle cerebral artery (STA-MCA). The EC-IC bypass study published in 1985 failed to demonstrate the efficacy of STA-MCA bypass over medical management in over 1,300 patients with occlusive disease of the anterior circulation. ${ }^{1}$ Patients with transient ischaemic attack (TIA) or stroke in the setting of occlusive disease not amenable to carotid endarterectomy (primarily carotid occlusion) were randomised to best medical therapy or best medical therapy in addition to EC-IC bypass. Although surgery was performed successfully, with a reported bypass patency rate of $96 \%$, EC-IC bypass conferred no benefit in terms of stroke risk over the average follow-up period of 55.8 months.

Subsequent analysis of the study methodology identified shortcomings in study design and implementation that suggested universal abandonment of EC-IC bypass for ischaemic was premature.,3 The trial was criticised for potential selection bias in enrolment, with concerns that a large number of patients, more than enrolled within the study, actually underwent surgery outside the trial. This would suggest that patients may have been selectively offered surgery versus enrolment in the trial based on perceived benefit of intervention, thus diluting any beneficial effect of bypass in those within the study cohort. Concern was also raised regarding the adequacy of flow augmentation provided by the STA-MCA bypass, with the notion that higher flows through larger calibre bypass could be more effective. The STA-MCA bypass has been the primary type of bypass performed in the setting of ischaemia, due to its high technical success rate and low morbidity given that anastomosis is performed to a distal cortical MCA branch with little risk of ischaemia during crossclamping. ${ }^{4}$ However, the degree of flow augmentation can be relatively modest compared with larger conduit bypasses such as vein or radial artery interposition grafts from the cervical carotid to the more proximal MCA. Larger conduits do carry several disadvantages, however, including higher morbidity and lower patency rates, ${ }^{5}$ and the risk of ischaemia during cross-clamping of the more proximal MCA. Additionally, there is concern for hyperperfusion haemorrhage when using high flow grafts to revascularise ischaemic brain, ${ }^{6}$ presumed to be due to the inability of the vasodilated ischaemic vascular bed to compensate acutely to a large increase in perfusion created by a high flow graft. Favourable preliminary results with higher flow grafts in small series have been reported with the use of a non-occlusive bypass technique, the excimer laser-assisted anastomosis (ELANA), which allows vein grafts to be placed onto large 
proximal vessels, such as the supraclinoid carotid artery or proximal MCA, without clamping the vessel during anastomosis. ${ }^{7.8}$ This technique is still under evaluation, but the lack of temporary vessel occlusion during surgery, or the more physiological placement of the bypass proximally in the intracranial circulation, appears to reduce the risk of hyperperfusion.

The most prominent drawback of the EC-IC bypass trial, which ultimately generated the most subsequent analysis and investigation, was the lack of haemodynamic evaluation in selecting patients for enrolment. In the trial, no objective physiological criteria were used to assess cerebral blood flow, and thus patients may have presented with ischaemic symptoms secondary to aetiologies unlikely to benefit from bypass, such as embolic phenomena or small vessel disease. Subsequent to the ECIC bypass trial, the role of haemodynamic assessment in evaluation of carotid occlusive disease has been extensively evaluated, demonstrating that haemodynamic evaluation can identify a subgroup at higher risk of recurrent stroke, ${ }^{9-15}$ and who would, therefore, be more appropriate candidates for revascularisation. In the last two decades, two trials have attempted to address the efficacy of bypass in such patients with haemodynamic compromise. The Japanese EC-IC bypass trial (JET) randomised patients with symptomatic internal carotid artery (ICA) or MCA occlusive and haemodynamic impairment to undergo STA-MCA bypass versus medical therapy. Interim results based on analysis of 196 patients published in a Japanese language journal reported a favourable result for bypass at two-year follow-up, ${ }^{16}$ with a reduced risk of the primary endpoint of death and stroke $(23.1 \%$ in the medical arm and $15.2 \%$ in the surgical arm; $\mathrm{p}=0.046$ ). The Carotid Occlusion Surgery Study (COSS) based in the US, however, did not support the efficacy of bypass for stroke-risk reduction. ${ }^{17}$

coss enrolled patients with symptomatic carotid occlusion and haemodynamic impairment based on evidence of severe compromise on positron emission tomography (PET), which was considered to be more relevant than blood flow measurement alone. ${ }^{18}$ The study compared STA-MCA bypass with medical therapy alone, with a primary endpoint of ipsilateral ischaemic stroke within 2 years, plus 30-day stroke and death. The study was prematurely halted in June 2010, after randomisation of only 193 of the originally planned 372 patients. The termination of the study was based on futility analysis, as Kaplan-Meier estimates of the primary endpoint demonstrated essentially equivalent rates for the medical and surgical groups, at $23 \%$ and $21 \%$, respectively. This occurred despite excellent graft patency rates and improvement in cerebral haemodynamics post bypass. The surgical group suffered a 30 day (peri-operative) event rate of $15 \%$, and despite a low subsequent stroke risk thereafter, failed to provide an overall benefit, given that the medical group actually demonstrated a much lower event rate than the $40 \%$ stroke risk originally projected based on prior observational data. ${ }^{14,19}$ Based on interim analysis, it was felt that a substantial increase in sample size from the originally anticipated 372 patients would be required to demonstrate any clinically meaningful difference in favour of surgery, and thus the study was terminated.

Although coss was a carefully designed and well-executed study, several aspects of the study deserve further consideration. ${ }^{20}$ The lower than anticipated stroke rate in the medical arm of the trial was largely attributed to improvements in medical therapy such as use of statins However, the magnitude of the reduction approaches or exceeds that seen in trials of intensive active medical management, ${ }^{21,22}$ and raises the potential concern that the criteria for selection of highest-risk patients into the trial failed to do so as effectively as planned. The use of a semi- quantitative method for assessment of the haemodynamic compromise, based on relative right to left ratio, rather than the original absolute threshold utilised in earlier observational studies, has been raised as a potential concern in appropriately selecting the most compromised individuals. ${ }^{23}$ Regardless, the surgical arm suffered a higher than desirable peri-operative event rate, at $15 \%$. Within the study, 30 surgeons performed the 93 bypasses, ${ }^{24}$ which generally reflects the low-volume nature of this operation at any given site. A volume-outcome relationship has been well demonstrated in many aspects of cerebrovascular surgery, including ECIC bypass, where the relationship has been found to be most robust when considering not only just individual surgeon caseload but also hospital volume. This may reflect the influence of epiphenomenon to the surgery including specialised nursing, anaesthesia and critical care in addition to the surgeon experience. If peri-operative event rates closer to $8 \%$ had been achieved, surgery would have demonstrated clear benefit in the coss population. It is worth noting that the stroke event rate after the 30 day peri-operative period was significantly lower in the bypass patients at $9 \%$ compared with $22.7 \%$ in the medical group. In conjunction with the haemodynamic improvement seen on PET in the surgical patients, these data do seem to confirm the general concept that revascularisation is beneficial, but only if it can be performed with low enough surgical risk. Even with the current rates reported in COSS, if stroke rates were to remain steady in both groups beyond the 2-year follow-up, the KaplanMeier curves would cross over to a significant benefit of surgery at about 5 years. One other important consideration when interpreting the results of coss is the recognition that haemodynamically unstable patients, although a small subgroup of patients with carotid occlusion, were not well represented in the study population. The mean time to enrolment within COSS was 72 days, and thus likely represented more stable patients.

In conjunction with COSS, an ancillary study, Randomized Evaluation of Carotid Occlusion and Neurocognition (RECON), followed a subset of patients to examine cognitive outcomes. The results have not yet been published, but preliminary presentation of the data at the International Stroke Conference 2013 indicated that no cognitive benefit was realised in the small cohort studied. There has been prior non-randomised data supporting improvement of cognitive function, post bypass ${ }^{25,26}$ and even the interesting notion of functional benefits, such as increased brain volume and improvement in fixed neurological deficits. ${ }^{27-29}$ Such preliminary data will require further investigations.

After recent publication of the technical aspect of the COSS, ${ }^{24}$ several concerns have also been voiced from Japanese vascular neurosurgeons. First, the duration of MCA occlusion in the study might have been too long: in patients who developed ischaemic stroke, the average occlusion time was 55.9 minutes. ${ }^{24}$ Even in those who did not develop stroke, the duration was 45.4 minutes. ${ }^{24}$ Experienced Japanese neurosurgeons who routinely perform EC-IC bypass procedures typically report completing suturing of the STA to the MCA within 30 minutes of occlusion time. ${ }^{30,31}$ Although the list of participants in the coss trial included many expert vascular neurosurgeons, the longer average occlusion times may reflect the relative rarity of EC-IC bypass procedures in the US. Although occlusion times in COSS were not linked to risk of peri-operative stroke per se, there is lingering concern that this may have been a contributory factor. Additionally, a single anastomosis was performed in COSS patients, ${ }^{24}$ which may provide insufficient blood flow to the ischaemic area. Although elevated oxygen extraction fraction (OEF) was markedly improved post bypass; it did not normalise. By contrast, it is the Japanese practice to create a double anastomosis to the frontal and temporal branches of the middle cerebral artery. ${ }^{30,31}$ 
However, consideration should be made about possible mentioned increased risk of hyperperfusion syndrome and risk of skin necrosis and the fact that single bypass usually tends to increase flow volume during long follow-up. Moreover, the frontal branch may be within the collateral system of the occluded ICA via the ophthalmic artery. Second, there has been little description in the published study of how patients were managed post-operatively, except that they received oral aspirin.17,24 Japanese neurosurgeons are well aware that strict blood pressure control is essential for patients who undergo EC-IC bypass, since the incidence of hyperperfusion syndrome may not be rare. ${ }^{30,31}$ The use of near-infrared spectroscopy both for surgery and for non-invasive bedside measurement of regional cerebral oxygenation seems to be useful in the early detection of hyperperfusion syndrome. ${ }^{31}$ Interestingly, the great majority of reports on hyperperfusion syndrome have come from Asian countries, ${ }^{30,31}$ and it is uncertain whether the potential risk of hyperperfusion syndrome had been fully recognised by the neurocritical care physicians participating in coss. Japanese neurosurgeons also have to do more: although positive results for the surgical treatment have been obtained from the JET study, it has still not been published in peer-reviewed English-language journal at the time of writing, ${ }_{1}^{16}$ even though more than 10 years have passed after completion of the JET study. Medical treatment has improved significantly during that period, and new data would be required to convincingly demonstrate to neurologists, neurosurgeons and patients worldwide that EC-IC bypass surgery is truly efficacious and safe.

\section{Posterior Circulation}

Data regarding bypass for posterior circulation ischaemia are sparse. This likely reflects the relative prevalence of the condition, and the emergence of less-invasive endovascular techniques in the face of the relative higher morbidity and technical complexity of posterior circulation bypass. ${ }^{32,33}$ Furthermore, objective assessment of haemodynamic compromise in the posterior circulation has generally been more difficult: the imaging methods used in evaluation of anterior circulation ischaemia are less useful in assessment of the posterior circulation, ${ }^{34}$ due to the more compact brain territory and skull base artefacts. An alternative method for assessing posterior circulation blood flow relies on large vessel flow measurement using quantitative magnetic resonance angiography (QMRA), a technique utilising phase contrast MR to measure vessel specific volumetric flow rates. Preliminary results from patients with vertebrobasilar occlusive disease have shown that QMRA can identify patients with flow compromise, a group at higher risk of recurrent stroke. ${ }^{35}$ The utility of this imaging in identifying high-risk patients is currently under investigation in prospective fashion. ${ }^{36}$

Bypass operations utilising a variety of donor and recipient vessels have been described for the posterior circulation, including occipital artery (OA) to posterior inferior cerebellar artery (PICA), STA to posterior cerebral artery (PCA) and STA to superior cerebellar artery (SCA) bypasses. Overall, these carry higher risk and lower patency rates than those seen with anterior circulation STA-MCA bypass. Patency rates for OA-PICA bypass range from 88 to $100 \%$, with mortality averaging $4 \%{ }^{32}$ STA-PCA and STA-SCA bypasses carry a 78-90\% patency rate, with mortality averaging $12 \%{ }^{32,33}$ and serious morbidity of $20 \%$. Although these series report improvement in a subset of patients, the morbidity and mortality have introduced caution when considering bypass, particularly in patients with poor neurological condition or medical co-morbidities. Consequently, EC-IC bypass is performed rarely in this situation, and on a case-by-case basis reserved for the infrequent situation when symptoms are refractory to maximal medical therapy, haemodynamic compromise is demonstrable and the disease is not amenable to endovascular interventions.

\section{Current Indications for Extracranial- Intracranial Bypass}

Following publication of the original EC-IC bypass trial, surgical revascularisation with bypass in the setting of atherosclerotic disease has become increasingly restricted to a small, and select, population of patients who experience refractory symptoms despite maximal medical therapy. ${ }^{37,38}$ With the more recent trial data, EC-IC bypass cannot be considered a routine procedure for patients presenting with initial ischaemic event in the setting of atherosclerotic occlusive disease. However, selected patients with severe haemodynamic impairment and/or recurrent symptoms despite maximal medical therapy could benefit from surgery at high-volume centres that offer the procedure with low peri-operative morbidity.

\section{Angioplasty and Stenting for Intracranial Atherosclerotic Stenosis}

Intracranial atherosclerotic stenosis is a cause of 8 to $10 \%$ of cases of ischaemic stroke in the US and up to $33 \%$ of ischaemic strokes in Asia, respectively. ${ }^{39-41}$ The annual risk of stroke from all causes in patients with intracranial atherosclerosis is estimated to be $3.6 \%$ to $13 \%{ }^{42-49}$ Angioplasty and/or stenting are advocated to improve cerebral blood flow and stabilise vulnerable plaque, with a goal to prevent recurrent stroke in patients with symptomatic and severe intracranial stenosis. ${ }^{50}$

\section{Evidence for Angioplasty and Stenting}

Favourable results using self-expanding stents aroused interest and subsequently initiated studies into neurointerventional treatment of intracranial atherosclerotic stenosis worldwide. ${ }^{51}$ The risk of major periprocedural complication as represented by stroke or death at 30 days following intracranial stenosis using Wingspan varied from $4.5 \%$ to $9.6 \%$ in the early studies. ${ }^{51-53}$ Over a mean imaging follow-up time of 8.5 months, the incidence of in-stent restenosis (ISR) following Wingspan was reported to be $28.3 \%(36 / 127)$, with $3.9 \%(5 / 127)$ evolving into complete stent occlusion: a third of them were symptomatic. ${ }^{54}$

Stenting and Aggressive Medical Therapy for Preventing Recurrent Stroke in Intracranial Stenosis (SAMMPRIS) was an investigator-initiated randomised, controlled, multi-centre clinical trial to test whether early angioplasty and stenting can reduce subsequent ischaemic stroke risk in patients with symptomatic severe stenosis of a major intracranial artery. ${ }^{55}$ Medical management in SAMMPRIS was identical in the two groups and consisted of aspirin, at a dose of $325 \mathrm{mg}$ per day; clopidogrel, at a dose of $75 \mathrm{mg}$ per day for 90 days after enrolment; management of the primary risk factors (elevated systolic blood pressure and elevated low-density lipoprotein [LDL] cholesterol levels) and management of secondary risk factors (diabetes, elevated non-high-density lipoprotein [non-HDL] cholesterol levels, smoking, excess weight and insufficient exercise) were achieved with the help of an intensive medical regimen and a lifestyle modification programme. It was found that angioplasty and stenting was inferior to aggressive medical therapy in prevention of recurrent stroke in patients with intracranial atherosclerotic stenosis. The peri-procedural stroke or death rate within 30 days after Wingspan stenting $(14.7 \%)$ in SAMMPRIS was substantially higher than the rates reported in earlier case series. ${ }^{51-53}$

The high rate of peri-procedural complication was attributed to inclusion of patients with recent symptoms that could lead to increased risk of 
distal embolism during stenting. ${ }^{56,57}$ Peri-procedural complications in SAMMPRIS were mainly due to ischaemic stroke $(63.3 \%, 19 / 30)$, which was largely due to perforator stroke $(63.1 \%, 12 / 19)$, and others such as embolic, mixed perforator and embolic, and stent occlusion. The other peri-procedural complications were due to haemorrhagic stroke, which was either subarachnoid haemorrhage $(45.5 \%, 5 / 11)$ or intracerebral haemorrhage (IC) $(54.5 \%, 6 / 11)$. The majority $(83.3 \%, 5 / 6)$ of IC presented $\geq 4$ hours after stenting. All cases of fatal haemorrhage were due to IC..$^{58}$

The importance of a learning curve factor in intracranial stenting has been highlighted subsequently. A low peri-procedural complication rate of $5 \%$ was reported in a prospective study of 100 consecutive cases performed in China, ${ }^{59}$ and the low complication rate was attributed to the experience of the two operators, who had prior experience of more than 180 procedures of intracranial stenting. ${ }^{60} \mathrm{~A}$ learning curve seems to be necessary for mastering Wingspan stenting for intracranial atherosclerosis and preventing peri-procedural complications from occurring.

Another study from Hong Kong confirmed that the peri-procedural complication rate could be lower $(5.3 \%, 3 / 57)$ when the procedures were performed in a referral centre by a constant team of operators. ${ }^{.1}$ The incidence of ISR at 1-year follow-up with digital subtraction angiography (DSA) in this Hong Kong study was $16.7 \%(11 / 66){ }^{62}$ and all were asymptomatic. In subsequent radiological follow-up, luminal gain beyond baseline diameter was observed in 36 lesions (54.5\%). ${ }^{62}$

Alternatives to self-expanding stents have also attracted attention recently. There is a revival of interest in primary balloon angioplasty. ${ }^{63}$ Additionally, a 182-patient series of balloon-expandable stents in intracranial atherosclerotic disease was recently reported. ${ }^{64}$ Technical success was achieved in $97 \%$ and the incidence of stroke at 30 days was $5.6 \%$. The 1-year incidence of all strokes or TIAs (including periprocedural) in this study was 18 (9.8\%), and all these symptomatic patients were advised to continue dual antiplatelets lifelong.

\section{Current Indications for Angioplasty and Stenting}

With the results of SAMMPRIS, angioplasty and stenting with selfexpandable stents should not be employed for first-time acute ischaemic stroke related to intracranial atherosclerotic stenosis. Acceptable 30-day stroke and mortality rate may be achievable in carefully selected patients with recurrent stroke related to intracranial atherosclerotic stenosis and refractory to medical treatment. Whether a certain subgroup may benefit more from neurointervention should be further investigated. ${ }^{65}$ Different techniques such as primary balloon angioplasty, balloon expandable stents, drug-eluting balloon and stents should be further assessed in future comparative effectiveness studies.
1. Failure of extracranial-intracranial arterial bypass to reduce the risk of ischemic stroke. Results of an international randomized trial. The EC/IC Bypass Study Group, N Eng/ J Med 1985;313:1191-200.

2. Sundt TM, Jr, Was the international randomized trial of extracranial-intracranial arterial bypass representative of the population at risk?, N Eng/ J Med, 1987;316:814-6.

3. Goldring S, Zervas N, Langfitt T, The Extracranial-Intracranial Bypass Study. A report of the committee appointed by the American Association of Neurological Surgeons to examine the study, N Eng/ J Med, 1987;316:817-20.

4. Horn P, Scharf J, Pena-Tapia P, Vajkoczy P, Risk of intraoperative ischemia due to temporary vessel occlusion during standard extracranial-intracranial arterial bypass surgery, I Neurosurg. 2008;108:464-9.

5. Regli L, Piepgras DG, Hansen KK, Late patency of long saphenous vein bypass grafts to the anterior and posterior cerebral circulation, J Neurosurg, 1995;83:806-11.

6. Stiver SI, Ogilvy CS, Acute hyperperfusion syndrome complicating EC-IC bypass, J Neurol Neurosurg Psychiatry, 2002;73:88-9.

Langer DJ, Van Der Zwan A, Vajkoczy P et al. Excimer laserassisted nonocclusive anastomosis. An emerging technology for use in the creation of intracranial-intracranial and extracraniaintracranial cerebral bypass, Neurosurg Focus, 2008;24:E6.

8. Klijn CJ, Kappelle $\amalg$, van der Zwan A, et al., Excimer laserassisted high-flow extracranial/intracranial bypass in patients with symptomatic carotid artery occlusion at high risk of recurrent cerebral ischemia: safety and long-term outcome, Stroke, 2002;33:2451-8.

9. Kleiser B, Widder B, Course of carotid artery occlusions with impaired cerebrovascular reactivity, Stroke, 1992:23:171-4.

10. Kuroda S, Houkin K, Kamiyama H, et al., Long-term prognosis of medically treated patients with internal carotid or middle cerebral artery occlusion: can acetazolamide test predict it?, cerebral artery occlusion:

11. Ogasawara K, Ogawa A, Yoshimoto T, Cerebrovascular eactivity to acetazolamide and outcome in patients with symptomatic internal carotid or middle cerebral artery occlusion: a xenon-133 single-photon emission computed tomography study, Stroke, 2002;33:1857-62.

12. Vernieri F, Pasqualetti P, Passarelli F, et al., Outcome of carotid artery occlusion is predicted by cerebrovascular reactivity, Stroke, 1999;30:593-8.

13. Webster MW, Makaroun MS, Steed DL, et al., Compromised cerebral blood flow reactivity is a predictor of stroke in patients with symptomatic carotid artery occlusive disease, J Vasc surg 1995:21:338-44

14. Grubb RL Jr, Derdeyn CP, Fritsch SM, et al., Importance of hemodynamic factors in the prognosis of symptomatic carotid occlusion, JAMA, 1998;280:1055-60.

15. Yamauchi $H$, Fukuyama $H$, Nagahama $Y$, et al., Significance of increased oxygen extraction fraction in five-year prognosis increased oxygen extraction fraction in five-year prognos of major cerebral

16. Ogasawara K, Ogawa A, [JET study (Japanese EC-IC Bypass Trial)], Nippon Rinsho, 2006;64(Suppl. 7):524-7.

17. Powers WJ, Clarke WR, Grubb RL Jr, et al., Extracranialintracranial bypass surgery for stroke prevention in hemodynamic cerebral ischemia: the Carotid Occlusion Surgery Study randomized trial, JAMA, 2011:306:1983-92. 18. Grubb RL Jr, Powers WJ, Derdeyn CP, et al., The carotid occlusion surgery study, Neurosurg Focus, 2003;14:e9.

19. Derdeyn CP, Gage BF, Grubb RL, Jr, Powers WJ, Cost-effectiveness analysis of therapy for symptomatic carotid occlusion: PET screening before selective extracranial-to-intracranial bypass versus medical treatment, J NuCl Med, 2000;41:800-807.

20. Amin-Hanjani S, Barker FG, 2nd, Charbel FT, et al., Extracranialintracranial bypass for stroke-is this the end of the line or a bump in the road?, Neurosurgery, 2012;71:557-61.

21. Chimowitz MI, Lynn MJ, Derdeyn CP, et al., Stenting versus aggressive medical therapy for intracranial arterial stenosis, N Engl J Med, 2011;365:993-1003.

22. Amarenco P, Benavente $\mathrm{O}$, Goldstein $L B$, et al., Results of the Stroke Prevention by Aggressive Reduction in Cholesterol Levels (SPARCL) trial by stroke subtypes, Stroke, 2009;40:1405-9.

23. Carlson AP, Yonas H, Chang YF, Nemoto EM, Failure of cerebral hemodynamic selection in general or of specific positron emission tomography methodology?: Carotid Occlusion Surgery Study (COSS), Stroke, 2011;42:3637-9.

24. Grubb RL, Jr, Powers WJ, Clarke WR, et al., Surgical results of the Carotid Occlusion Surgery Study, J Neurosurg, 2013;118:25-33. 25. Fiedler J, Priban V Skoda O, et al., Cognitive outcome after EC-IC bypass surgery in hemodynamic cerebral ischemia, Acta EC-IC bypass surgery in hemodynamic (Wien), 2011;153:1303-11; discussion 1311-12.

Neurochir (Wien), 2011;153:1303-11; discussion 1311-12.
26. Sasoh M, Ogasawara K, Kuroda K, et al., Effects of EC-IC bypass surgery on cognitive impairment in patients with hemodynamic cerebral ischemia, Surg Neurol, 2003;59:455-60.

27. Horn P, Scharf J, Pena-Tapia P, Vajkoczy P, Risk of intraoperative ischemia due to temporary vessel occlusion during standard extracranial-intracranial arterial bypass surgery, J Neurosurg, 2008;108:464-9.

28. Fierstra J, Maclean DB, Fisher JA, et al., Surgical revascularization reverses cerebral cortical thinning in patients with severe cerebrovascular steno-occlusive disease, Stroke, 2011:42:1631-7.

29. Inoue T, Jinnouchi J, Changes in brain volume after EC-IC bypass surgery, Acta Neurochir Suppl, 2008:103:79-82.

30. Ogasawara K, Komoribayashi N Kobayashi M, et al., Neura damage caused by cerebral hyperperfusion after arterial damage caused by cerebral hyperperfusion after arterial
bypass surgery in a patient with moyamoya disease: case bypass surgery in a patient with moyamo
report, Neurosurgery, 2005;56:E1380.

report, Neurosurgery, 2005;56:E1380.
31. Nakagawa A, Fujimura M, Arafune T, et al., Clinical implications of intraoperative infrared brain surface monitoring during superficial temporal artery-middle cerebral artery anastomosis in patients with moyamoya disease, J Neurosurg, 2009;111:1158-64

32. Hopkins LN, Budny JL, Complications of intracranial bypass for vertebrobasilar insufficiency, I Neurosurg, 1989:70:207-11.

33. Ausman II, Diaz FG, Vacca DF, Sadasivan B, Superficial temporal and occipital artery bypass pedicles to superior, anterior inferior, and posterior inferior cerebellar arteries fo vertebrobasilar insufficiency, J Neurosurg, 1990;72:554-8.

34. Haase J, Magnussen IB, Ogilvy CS, et al. Evaluating patients with vertebrobasilar transient ischemic attacks, Surg Neurol, 1999:52:386-92.

35. Amin-Hanjani S, Du X, Zhao M, et al., Use of quantitative magnetic resonance angiography to stratify stroke risk in symptomatic vertebrobasilar disease, Stroke, 2005;36:1140-45. 36. Amin-Hanjani S, Rose-Finnell L, Richardson D, et al., Vertebrobasilar Flow Evaluation and Risk of Transient Ischaemic Attack and Stroke study (VERITAS): rationale and design, Int J Stroke, 2010;5:499-505.

37. Tummala RP, Chu RM, Nussbaum ES, Extracranial-intracranial bypass for symptomatic occlusive cerebrovascular disease not amenable to carotid endarterectomy, Neurosurg Focus, 2003:14:e8

38. Amin-Hanjani S, Charbel FT, Is extracranial-intracranial bypass surgery effective in certain patients? Neurosurg Clin N Am, 2008;19:477-87.

39. Sacco RL, Kargman DE, Gu Q, et al., Race-ethnicity and determinants of intracranial atherosclerotic cerebral infarction Stroke, 1995;26:14-20.

40. Broderick J, Brott T, Kothari R, et al., The Greater Cincinnati/ Northern Kentucky Stroke Study: preliminary first-ever and total incidence rates of stroke among blacks, Stroke, 1998;29:415-21.

41. Wong KS, Huang YN, Gao S, et al., Intracranial stenosis in Chinese patients with acute stroke, Neurology, 1998;50:812-13. 42. Bogousslavsky J, Barnett HJ, Fox AJ, et al., Atherosclerotic disease of the middle cerebral artery, Stroke, 1986;17:1112-20.

43. Chimowitz MI, Kokkinos J, Strong J, et al., The WarfarinAspirin symptomatic intracranial disease study, Neurology, 1995:45:1488-93.

44. Thijs VN, Albers GW, Symptomatic intracranial atherosclerosis: Outcome of patients who fail antithrombotic therapy Neurology, 2000;55:490-97.

45. Chimowitz MI, Lynn MJ, Howlett-Smith $\mathrm{H}$, et al., Comparison of warfarin and aspirin for symptomatic intracranial arterial stenosis, N Engl J Med, 2005;352:1305-16

46. The EC/IC Bypass Study Group, Failure of extracranialintracranial arterial bypass to reduce the risk of ischemic stroke (results of an international randomized trial), N Engl J Med, 1985;313:1191-200

47. Rundek T, Elkind MS, Chen X, Increased early stroke recurrence among patients with extracranial and intracranial atherosclerosis: Northern Manhattan Stroke Study, Neurology, 1998;50(Suppl. 4):A75.

48. The Warfarin-Aspirin Symptomatic Intracranial Disease (WASID) Study Group. Prognosis of patients with symptomatic vertebral or basilar artery stenosis, Stroke, 1998:29:1389-92.

49. Caplan LR, Advances in stroke research: Basic science, treatment and clinical trial outcomes, Rev Neurol Dis, 2004;2:91-4.

50. Qureshi Al, Feldmann E, Gomez CR, et al., Consensus conference on intracranial atherosclerotic disease: rationale, methodology, and results, J Neuroimaging, 2009;19:1S-10S

51. Bose A, Hartmann M, Henkes H, et al., A novel, self-expanding, nitinol stent in medically refractory intracranial atherosclerotic stenoses: the Wingspan study, Stroke, 2007;38:1531-7.

52. Fiorella D, Levy El, Turk AS, et al., US multicenter experience with the wingspan stent system for the treatment of intracranial atheromatous disease: periprocedural results, Stroke, 2007;38:881-7.

53. Zaidat OO, Klucznik R, Alexander MJ, et al., NIH Multi-center Wingspan Intracranial Stent Registry Study Group. The $\mathrm{NIH}$ registry on use of the Wingspan stent for symptomatic 70-99\% intracranial arterial stenosis, Neurology, 2008:70:1518-24.

54. Albuquerque FC, Levy EI, Turk AS, et al., Angiographic patterns of Wingspan in-stent restenosis, Neurosurgery, 2008;63:23-7.

55. Chimowitz MI, Lynn MJ, Derdeyn CP, et al., Stenting versus Aggressive medical therapy for intracranial arterial stenosis, N Eng/ J Med, 2011;365:993-1003.

56. Gray WA, Yadav IS, Verta P, et al., The CAPTURE registry: predictors of outcomes in carotid artery stenting with embolic protection for high surgical risk patients in the early post-approval setting Catheter Cardiovasc Interv, 
2007;70:1025-33.

57. Topakian R, Strasak AM, Sonnberger M, et al., Timing of stenting of symptomatic carotid stenosis is predictive of 30 day outcome, Eur J Neurol, 2007;14:672-8.

58. Fiorella D, Derdeyn CP, Lynn MJ, et al., Detailed Analysis of Periprocedural Strokes in Patients Undergoing Intracranial Stenting in Stenting and Aggressive Medical Management for Preventing Recurrent Stroke in Intracranial Stenosis (SAMMPRIS), Stroke, 2012;43:2682-16888.

59. Jiang WJ, Yu W, Du B, Gao F, Cui LY, Outcome of patients with 9. Jiang WJ, Yu W, Du B, Gao F, Cui LY, Outcome of patients with
$\geq 70 \%$ symptomatic intracranial stenosis after wingspan stenting, Stroke, 2011;42:1971-5.

60. Jiang WJ, Du B, Leung TW, Xu XT, et al., Symptomatic intracranial stenosis: cerebrovascular complications from elective stent placement, Radiology, 2007;243:188-97.

61. Yu SC, Leung TW, Hung EH, et al., Angioplasty and stenting for intracranial atherosclerotic stenosis with nitinol stent: factors affecting technical success and patient safety, Neurosurgery, 2012;70(1 Suppl. Operative):104-13.

62. Yu CH, Leung WH, Hung HY, et al., Angioplasty and stenting of intracrani a intracranial atherosclerosis with Wingspan system evaluation
of factors affecting one-year restenosis of 66 cases in a single center. 97th Scientific Assembly and Annual Meeting of the RSNA 2011, Chicago, USA, 27.

63. Tomycz L, Bansal NK, Lockney T, et al., Primary balloon angioplasty for symptomatic, high-grade intracranial stenosis, Surg Neurol Int, 2013; 4:18.

64. Alkukar A, Karanam LS, Oak S, et al., Role of balloon-

expandable stents in intracranial atherosclerotic disease in a series of 182 patients, Stroke, 2013;44:2000-2003.

65. Wong GK, Poon WS, Time to reflect on surgery and neuroWong GK, Poon WS, Time to reflect on surgery and neurointervention for intracran 